\title{
A convolution of observational and model data to estimate age of air spectra in the northern hemispheric lower stratosphere
}

Marius Hauck et al.

Correspondence to: Marius Hauck (hauck@iau.uni-frankfurt.de)

The copyright of individual parts of the supplement might differ from the CC BY 4.0 License. 

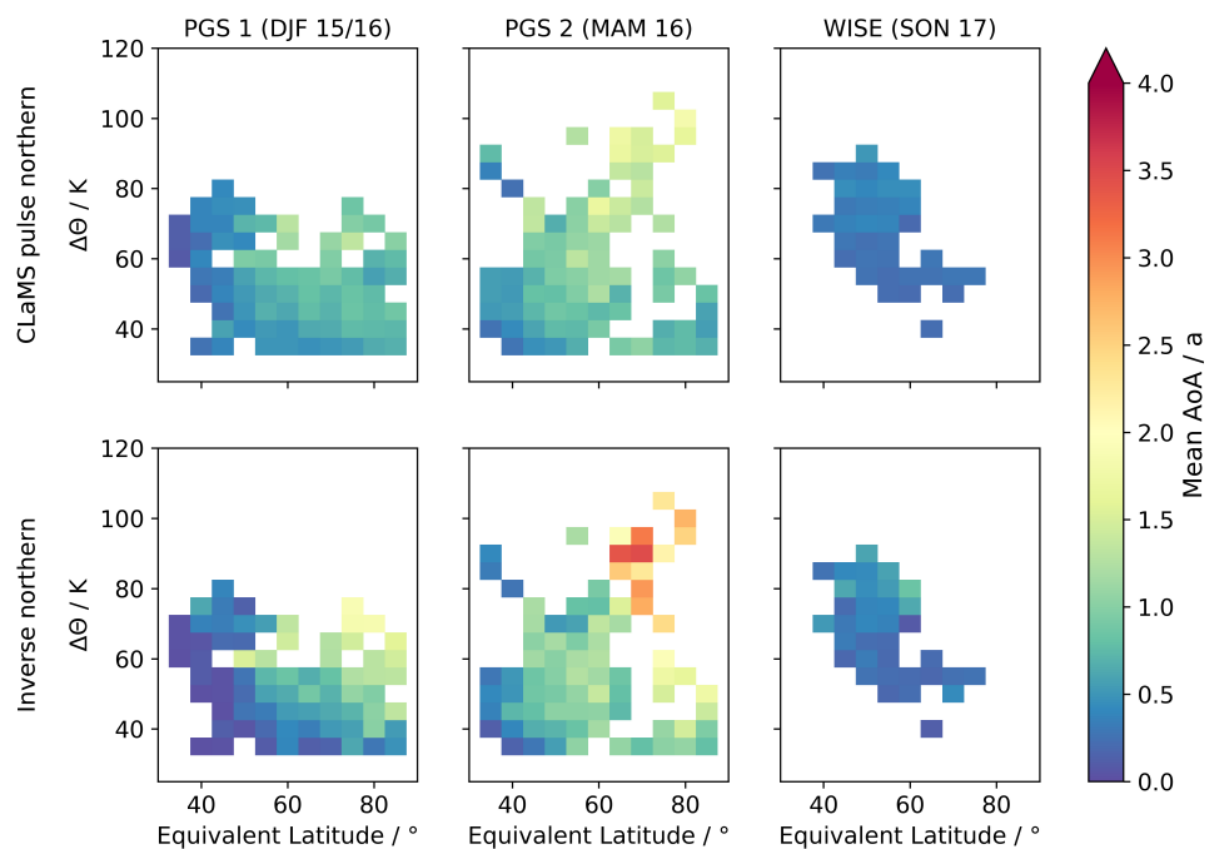

Figure S1: Cross sections of binned mean AoA calculated using the normalized age spectra referring to the northern hemispheric tropopause 5 section $G_{N}$ from the CLaMS pulse tracers (top row) and again from the inverse method in the Monte Carlo simulation using observational data (bottom row - same as in Fig. 6 to ease comparison). Left column shows data of PGS phase 1, mid column data of PGS phase 2 and right column data of WISE. The potential temperature difference to the local tropopause $\Delta \Theta$ is used as vertical coordinate, equivalent latitude as horizontal coordinate. 

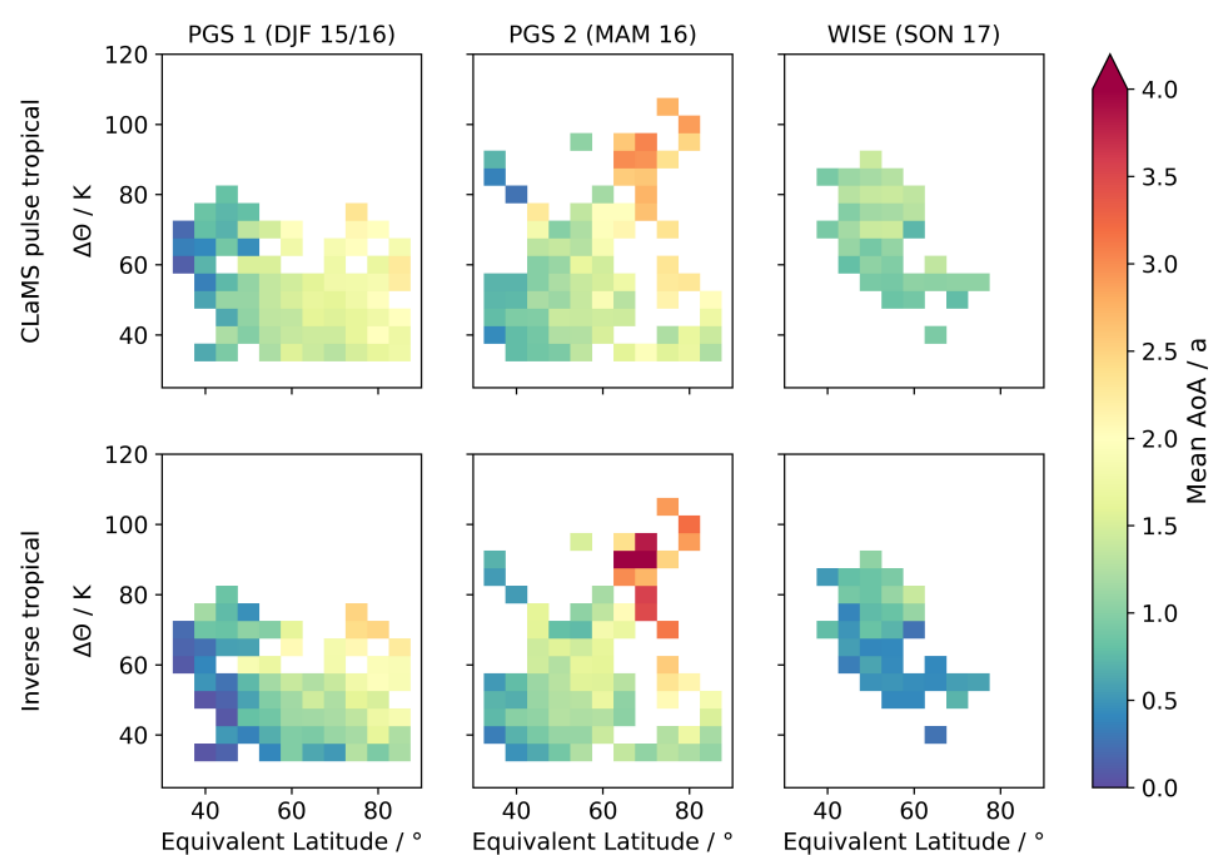

Figure S2: Same as Fig. S1, but for age spectra with reference at the tropical tropopause $G_{T}$.

5 

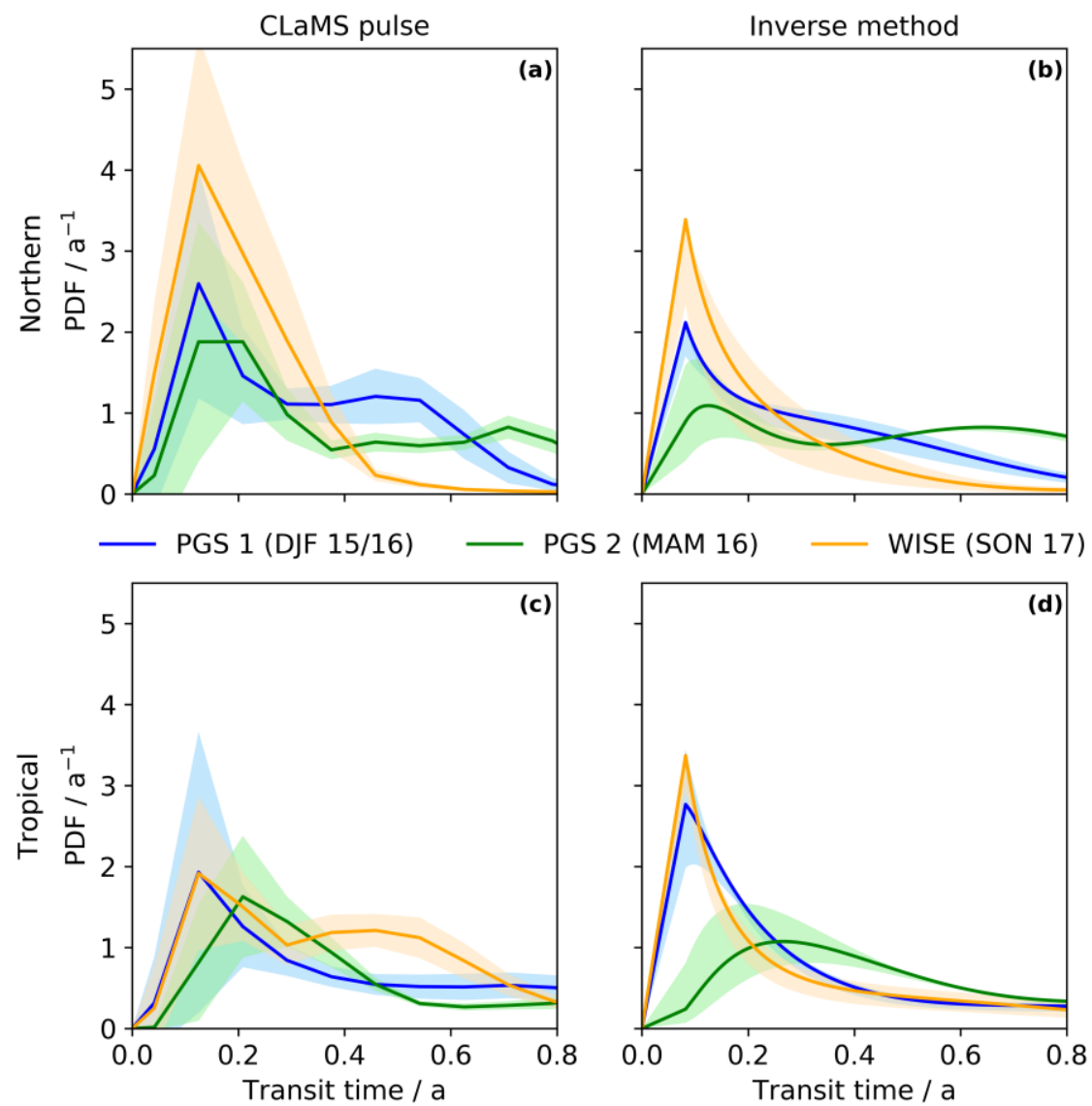

Figure S3: Campaign-averaged age spectra for PGS phase 1 (blue), PGS phase 2 (green) and WISE (orange). Top row displays normalized age spectra with reference at the northern hemispheric tropopause section $G_{N}$ from CLaMS pulse tracers (panel (a)) and inverse method (panel (b)) using observational data (same as in Fig. 7 to ease comparison). Bottom row shows normalized age spectra with origin at the tropical tropopause $G_{T}$ from CLaMS pulse tracers (panel (c)) and inverse method (panel (d)) using observational data. Colored shadings give the uncertainty range of the spectra derived in the binning procedure for CLaMS pulse spectra and in the Monte Carlo simulation for the inverse spectra (see Sect. 2.2.4 for details). Note that transit times smaller than one month have been excluded from the inverse spectra to keep them comparable with CLaMS pulse age spectra that are limited to a monthly resolution in transit time for the shown range. 

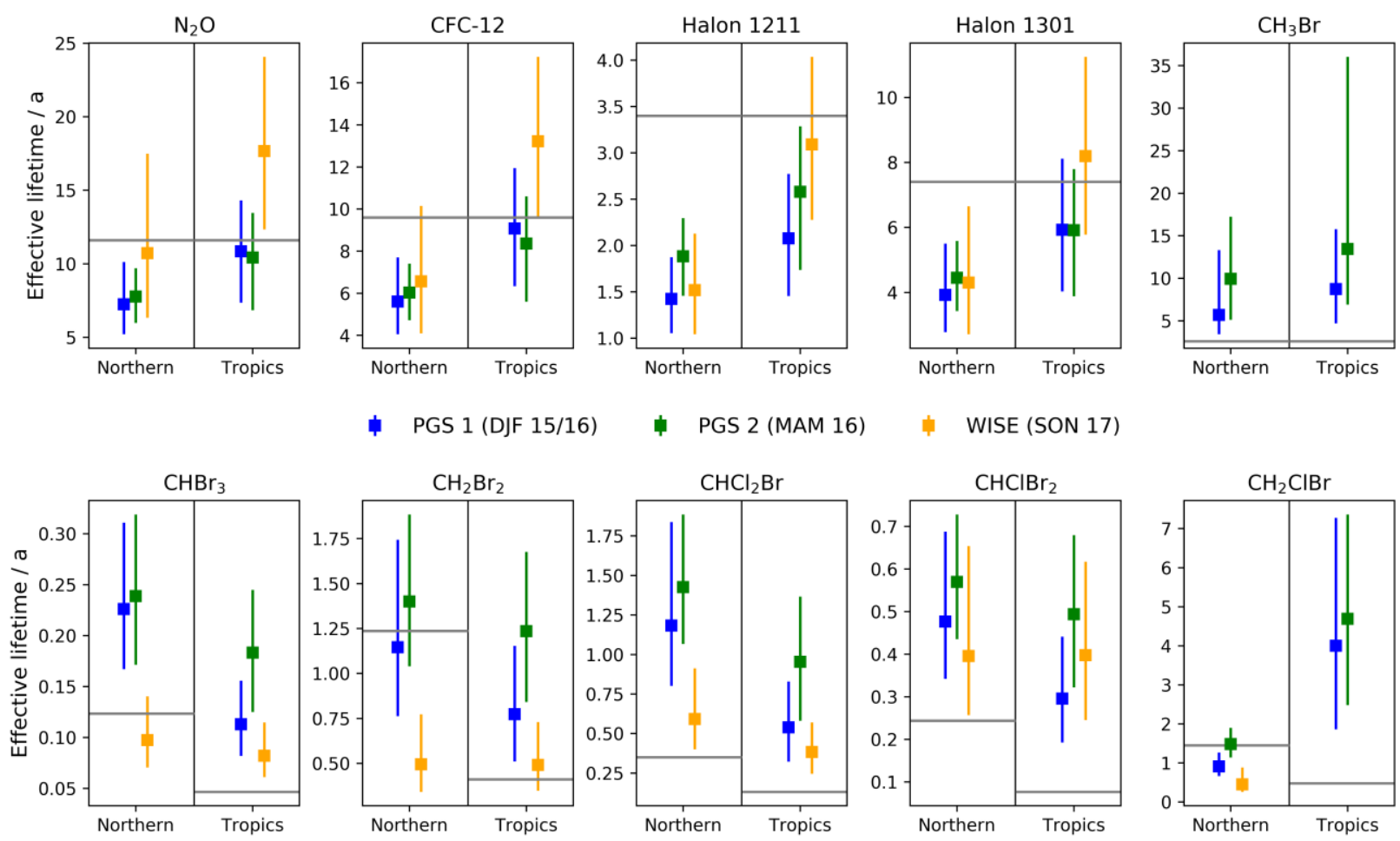

Figure S4: Retrieved effective lifetimes of the campaign-averaged inverse age spectra for PGS phase 1 (blue), PGS phase 2 (green) and

5 WISE (orange). Left sections in each panel refers to age spectra with origin at the northern hemispheric tropopause region, while the right sections refer to age spectra with origin at the tropical tropopause. Grey horizontal lines show the initial guess for each lifetime used in the retrieval procedure. Error bars denote the derived uncertainty from the Monte Carlo simulation. Note that missing values indicate that no effective lifetime could be retrieved with the Monte Carlo simulation, so that the substance has been omitted. 